\title{
Analyse freier Schriftproduktionen als Instrument für die Einstufung von Studierenden mit russischsprachigem Hintergrund
}

\author{
Natalia Ermakova (Potsdam)
}

В статье речь идет об особенностях и сложностях проведения вступительного тестирования ААя немецкоговорящих студентов, освоивших русский язык в семье и готовящихся к изучению русского языка в рамках одного из филологических направлений, преАлагаемых в ПотсАамском Университете (Германия). Многолетний опыт работы с данной категорией учащихся показац, что одним из наиболее эффективных инструментов оценки их знаний по русскому языку явАяется анализ написанных ими сочиненийописаний. В статье преАлагается набор критериев, объединяющих знания текстологии, методики русского как родного и РКИ, Аля оценки этих работ. Кроме того, подчеркивается важность применения описанных критериев не только Аля обнаруживания и систематизации ошибок в письменных работах учащихся, но и Аля распознания уже имеющихся у них знаний, которые Аолжны послужить отправной точкой Аля планирования занятий по русскому языку в данной аудитории.

\section{1. $\quad$ Einleitung}

Es ist eine unbestreitbare Tatsache, dass jede sprachförderliche Handlung von Lehrenden auf den Vorkenntnissen der Lernenden aufbauen muss. Jedoch ist die Einschätzung dieser Vorkenntnisse nicht immer einfach und sie stellt Lehrkräfte vor viele Herausforderungen. Besonders komplex und kompliziert ist die Erfassung des Sprachstandes bei Lernenden mit muttersprachlichem Hintergrund.

Am Zentrum für Sprachen und Schlüsselkompetenzen (Zessko) der Universität Potsdam ist in den letzten Jahren folgende Situation zum Alltag geworden:

Anka Bergmann, Olga Caspers \& Wolfgang Stadler (Hg.)

Didaktik der slawischen Sprachen - Beiträge zum 1. Arbeitskreis in Berlin (12.-14.9.2016)

(C) 2018 innsbruck university press, ISBN 978-3-903187-11-5, DOI 10.15203/3187-11-5 
Fast 60\% der StudienanfängerInnen, die sich für einen philologischen Studiengang entscheiden und am sprachpraktischen Russischunterricht teilnehmen müssen, verfügen über einen sog. russischsprachigen Hintergrund. Die meisten dieser Studierenden sind VertreterInnen der zweiten MigrantInnengeneration. Dies bedeutet, dass sie mit ihren Familienangehörigen aus verschiedenen Gründen im Vorschulalter nach Deutschland kamen ${ }^{1}$ und Russisch bereits als Erstsprache erworben, jedoch keinen institutionellen Russischunterricht erlebt haben (vgl. Anstatt 2011, s. auch Brüggemann in diesem Sammelband). Bei diesen Studierenden sind gravierende Differenzen in unterschiedlichen Sprachbereichen zu beobachten. So können sie meist akzentfrei und sicher in Alltagssituationen kommunizieren, haben einen reichen Wortschatz, der einerseits viele Themen abdeckt, andererseits sehr lückenhaft ist. Viele der Studierenden können entweder gar nicht oder nur in geringem Ausmaß lesen und schreiben. Im schriftlichen Bereich werden der unvollständige Grammatikerwerb (Brüggemann 2014) und der fehlende Bezug zwischen gesprochenem und geschriebenem Russisch besonders sichtbar. Trotz der genannten Problematik werden diese Studierenden in vielen Spracheinrichtungen nach wie vor als MuttersprachlerInnen definiert und vom sprachpraktischen Unterricht befreit. Diese Tendenz lässt in jüngster Zeit jedoch immer mehr nach und KollegInnen werden aus diesem Grund immer öfter mit der Frage konfrontiert, wie der Sprachstand solcher HerkunftssprecherInnen effizient erhoben werden kann, um sie in den richtigen Sprachkurs einzustufen.

Wie Reich (2009: 25) treffend anmerkt, werden „Sprachdiagnose und Sprachförderung in der Regel je für sich bearbeitet, von verschiedenen Verfassern und nach ihrer je eigenen Logik“. Die zahlreichen Arbeiten aus den Gebieten der Soziolinguistik (Meng 2001, Rethage 2012), Kontaktlinguistik (Anstatt 2008), Linguistik (Anstatt 2009, 2010, Böhmer 2015, Dieser 2013, Karl 2012) und Didaktik (Bergmann 2014, Brüggemann 2010, 2012, 2014), die sich der oben beschriebenen Zielgruppe widmen, liefern wichtige Erkenntnisse, die für sprachförderliche Aktivitäten von großer Bedeutung sind. Wie diese Erkenntnisse um-

1 Laut den Daten aus einer über vier Jahre intern durchgeführten Fragebogenstudie liegt das Durchschnittsalter der Studierenden, die zum sprachpraktischen Russischunterricht ins Zessko kommen, bei der Ausreise nach Deutschland bei sieben Jahren.

Anka Bergmann, Olga Caspers \& Wolfgang Stadler (Hg.)

Didaktik der slawischen Sprachen - Beiträge zum 1. Arbeitskreis in Berlin (12.-14.9.2016)

(C) 2018 innsbruck university press, ISBN 978-3-903187-11-5, DOI 10.15203/3187-11-5 
gesetzt werden, bleibt jedoch meist der einzelnen Lehrkraft überlassen. Besonders was die Sprachdiagnose betrifft, liefern die genannten Studien viele allgemeine Informationen zu den sprachlichen Besonderheiten der Zielgruppe, bieten aber keine Anleitung für das Einstufungsverfahren jeder/jedes einzelnen Studierenden. Somit erarbeitet jede Spracheinrichtung die Einstufungstests ,für sich` und ,nach eigener Logik'.

Ziel dieses Artikels ist es, das Einstufungsverfahren am Zessko der Universität Potsdam in allgemeinen Zügen zu präsentieren sowie ein Instrument der Einstufung vorzustellen, welches sich für unsere Zwecke als besonders effizient erwiesen hat.

\section{Das Einstufungsverfahren am Zessko der Universität Potsdam}

Der diagnostische Einstufungstest, der von MitarbeiterInnen des Bereichs Slawische Sprachen am Zessko zur Optimierung der Einstufung neuer Studierender entwickelt wurde, dient folgenden Zwecken:

1. Einstufung von Studierenden der Studiengänge „BA Russistik“, „BA Russisch Lehramt“, „BA Interdisziplinäre Russlandstudien“ mit dem Ziel, das in den Studienordnungen als Eingangsvoraussetzungen festgelegte Sprachniveau der KursteilnehmerInnen zu ermitteln,

2. Ermittlung des Sprachniveaus nach GeR (2001) für Studierende aller Fakultäten.

Der Einstufungstest besteht aus drei Teilen bzw. verläuft in drei Phasen:

1. Schriftliche Bildbeschreibung (Verfassen eines Aufsatzes),

2. Online-Test in den Bereichen Grammatik, Lexik, Lese- und Hörverstehen,

3. Mündliches Interview in Form eines persönlichen Gesprächs mit jedem/ jeder Studierenden. 
Im Falle der Studierenden, die Russisch als Fremdsprache gelernt haben, sind der Online-Test und das Interview die Hauptinstrumente, die eine sehr genaue Einschätzung der Vorkenntnisse gewährleisten. Bei der Erfassung des Sprachstandes von Studierenden mit russischsprachigem Hintergrund haben sich diese beiden Instrumente jedoch als ungenau und unzureichend erwiesen. Grund dafür ist die ungleichmäßige sprachliche Entwicklung der Studierenden auf unterschiedlichen Sprachebenen. Dementsprechend zeigen sie während des Interviews gute mündliche Kompetenzen, die sich gravierend von ihren Fähigkeiten und Fertigkeiten im schriftlichen Bereich unterscheiden. Diese Studierenden verfügen außerdem oft über eine gute strategische Kompetenz. Dies bedeutet, dass sie während des Gesprächs geschickt die sprachlichen Strukturen, die sie nicht sicher genug beherrschen, vermeiden, sie umgehen oder kompensieren (können). Dadurch werden die Kenntnisse der Studierenden mit russischsprachigem Hintergrund bisweilen zu hoch eingeschätzt.

Während des Online-Tests ist häufig die umgekehrte Situation zu beobachten: Für die erfolgreiche Absolvierung dieses Tests ist die Beherrschung des kyrillischen Alphabets eine Voraussetzung, die diese Studierenden manchmal gar nicht oder nicht genügend erfüllen. Dementsprechend schneiden sie in diesem Teil des Einstufungstests, der großteils aus geschlossenen Fragen besteht, oft schlechter $\mathrm{ab}$, als es ihre mündlichen Kenntnisse erwarten lassen. So ist die Tatsache, dass sie in jenem Teil des Tests, der die Grammatikkenntnisse prüft, falsche Endungen wählen oder eintippen, nicht unbedingt damit verbunden, dass sie diese Endungen nicht kennen, sondern oft damit, dass ihnen der Bezug zwischen Graphem und Laut fehlt (unbetonte Vokale werden je nach Reduktionsstufe anders geschrieben als ausgesprochen).

Diese Tatsache spricht auch gegen die Anwendung des C-Tests, der gelegentlich an anderen Spracheinrichtungen ${ }^{2}$ zur Einstufung von Studierenden angewendet wird. Im Rahmen dieses Tests können die Studierenden mit russischsprachigem Hintergrund meistens die gekürzten Lexeme richtig erkennen, sie tippen die Antworten aber orthographisch fehlerhaft in die Lücken ein, was automatisch als falsche Lösung identifiziert wird und das Gesamtergebnis des Einstufungstests ver-

2 Z. B. am Sprachenzentrum der Universität Bochum: http://tinyurl.com/y7o5ddop [02.12.2017].

Anka Bergmann, Olga Caspers \& Wolfgang Stadler (Hg.)

Didaktik der slawischen Sprachen - Beiträge zum 1. Arbeitskreis in Berlin (12.-14.9.2016)

(C) 2018 innsbruck university press, ISBN 978-3-903187-11-5, DOI 10.15203/3187-11-5 
fälscht.

Die oben beschriebenen Schwierigkeiten der Anwendung von Online-Tests sowie die Ungenauigkeit der Einstufung von Kenntnissen bei Studierenden mit russischsprachigem Hintergrund nach Ergebnissen eines mündlichen Interviews sind jedoch kein Grund, um diese Instrumente der Sprachstandserhebung als absolut unanwendbar zu proklamieren und sie aus dem Einstufungsverfahren endgültig auszuschließen. Vielmehr müssen sie überdacht, optimiert und weiterentwickelt werden. Dies benötigt Zeit und weitere Forschung im Bereich der Leistungsbeurteilung von HerkunftssprecherInnen. Die tägliche Arbeit der Lehrkräfte kann jedoch nicht darauf warten, sie muss fortgesetzt werden. Aus diesem Grund ist eine Kombination mehrerer, einander ergänzender Instrumente zur Validierung der Ergebnisse empfehlenswert.

So werden am Zessko der Universität Potsdam der Online-Test und das mündliche Interview durch eine freie Schriftproduktion, konkreter durch eine Bildbeschreibung, ergänzt. Dieses Einstufungsinstrument hat sich, wie bereits oben angedeutet, bei der Sprachstandserhebung von Studierenden mit russischsprachigem Hintergrund als besonders aussagekräftig erwiesen.

\section{Freie Schriftproduktionen als Einstufungsinstrument}

Für die Elizitierung einer freien schriftlichen Textproduktion werden im Rahmen des Einstufungstests am Zessko der Universität Potsdam den teilnehmenden Studierenden verschiedene Bilder angeboten, die alltägliche Situationen darstellen und über viele Details verfügen. Für die Beschreibung eines gewählten Bildes haben die TeilnehmerInnen 20 Minuten Zeit. Form, Länge, Inhalt, Stil und Komplexität des Textes werden nicht durch Vorgaben gelenkt.

An dieser Stelle muss angemerkt werden, dass die Textsorte „Bildbeschreibung“ nicht willkürlich als Quelle einer freien Schriftprobe gewählt wird. Wie o. g., erhalten die Studierenden nur ein Bild als Ausgangspunkt für ihre Schreibproduktion und sind in ihrem Schreibprozess ansonsten völlig frei. Es muss jedoch hinzugefügt werden, dass eine Beschreibung für die Studierenden keine neue und unbekannte Textsorte darstellt. Sie kennen sie bereits aus dem schulischen

Anka Bergmann, Olga Caspers \& Wolfgang Stadler (Hg.)

Didaktik der slawischen Sprachen - Beiträge zum 1. Arbeitskreis in Berlin (12.-14.9.2016)

(C) 2018 innsbruck university press, ISBN 978-3-903187-11-5, DOI 10.15203/3187-11-5 
Deutschunterricht (vgl. Böhmer in diesem Sammelband). Diese Textsorte stellt an sie geringere Anforderungen als z. B. ein Bericht oder eine Erzählung, sie ist mit vielen anderen Textsorten verwandt und hat nur ein zentrales kommunikatives Ziel: den Leser dazu zu bringen, sich das Beschriebene genau vorzustellen (Fix 2008: 99-106). Mit welchen sprachlichen Mitteln, in welcher Form und in welchem Umfang das gemacht wird, ist dabei dem/der Schreibenden allein überlassen. Somit entstehen sehr unterschiedliche und schwer vergleichbare Schriftproduktionen (von zwei bis drei Sätzen bis zu zwei Seiten lang, unterschiedlich komplex, in kyrillischen, aber auch in lateinischen Buchstaben verfasst), die sowohl den Sprachstand der ProbandInnen auf unterschiedlichen sprachlichen Ebenen widerspiegeln, als auch ihre Kompetenz, mit den zur Verfügung stehenden sprachlichen Mitteln flexibel umzugehen, aufzeigen. Auf Grundlage dieser Textproben werden verschiedene Aspekte der sprachlich-kommunikativen Vorkenntnisse erhoben und eingeschätzt. Es muss hierbei auch erwähnt werden, dass derart unterschiedliche Texte unmöglich miteinander verglichen werden können. Bei ihrer Auswertung geht es auch nicht um eine Beurteilung nach Noten, sondern um die Einschätzung und Analyse der vorhandenen Kenntnisse eines/einer jeden Einzelnen.

Wie Schründer-Lenzen \& Henn (2009: 91) treffend anmerken, sind Textproduktionen „immer das Ergebnis sehr komplexer Prozesse. Sie stehen in Abhängigkeit von Welt- und Textmusterwissen, von Lexik und Syntax, von Planungsund Überarbeitungsstrategien und einer spezifischen literalen Kompetenz, die es einem/einer SchreiberIn ermöglicht, einen Text als Ganzes kohärent zu gestalten“. Die Analyse eines solchen Textes muss daher einerseits diesen Kriterien entsprechen, andererseits einen gewissen Freiraum bei der Bewertung lassen, da sich die sprachliche Flexibilität des/der AutorIn schwer nach festen Parametern einschätzen lässt.

\subsection{Kriterien für die Analyse freier Schriftproduktionen}

Bei der Einstufung von Studierenden arbeiten die Lehrkräfte unter komplizierten Umständen: Einerseits müssen sie in relativ kurzer Zeit die Sprachkenntnisse von vielen TeilnehmerInnen einschätzen, andererseits müssen sie bei jedem/jeder

Anka Bergmann, Olga Caspers \& Wolfgang Stadler (Hg.)

Didaktik der slawischen Sprachen - Beiträge zum 1. Arbeitskreis in Berlin (12.-14.9.2016)

(C) 2018 innsbruck university press, ISBN 978-3-903187-11-5, DOI 10.15203/3187-11-5 
einzelnen Studierenden individuell vorgehen. Die hohe StudentInnenzahl und die zeitliche Knappheit in einer solchen Situation miteinander zu verbinden, verlangt von Lehrkräften viel Geschick und Erfahrung. Ein vorliegendes Schema der Herangehensweise, eine Art Check-Liste, könnte jedoch dabei hilfreich sein. Im Weiteren wird ein Entwurf für solch eine Vorgehensweise präsentiert und anhand einiger konkreter Beispiele erläutert. Dieser Vorschlag sowie die beschriebenen Kriterien basieren auf der langjährigen Erfahrung der Autorin und dem Austausch mit Kolleginnen des Zessko, mit KollegInnen anderer Spracheinrichtungen im Rahmen unterschiedlicher Fortbildungen sowie auf eigenen Forschungsarbeiten zu den sprachlich-kommunikativen Voraussetzungen von Studierenden mit russischsprachigem Hintergrund (s. dazu z. B. Ermakova 2014, 2015, 2016).

\subsubsection{Erster Schritt - Metadaten erheben}

Um die Sprachkompetenz auf Basis der eingereichten Texte richtig einschätzen zu können, braucht die Lehrkraft einige Informationen zur Sprach(lern)biografie der Studierenden: In welchem Alter kam er/sie nach Deutschland? Was ist die Familiensprache? In welchen Situationen verwendet er/sie das Russische? Hat er/sie die Sprache je im gesteuerten Kontext gelernt (z. B. in der Schule oder im Rahmen eines Sprachkurses)? Das Erheben dieser Daten kann sowohl schriftlich (durch einen Fragebogen) als auch mündlich (durch ein kurzes Interview) erfolgen und der Lehrkraft helfen, manche sprachlichen Erscheinungen besser zu verstehen und richtig zu analysieren. Nach unseren Beobachtungen gilt, dass je niedriger das Einreisealter, desto höher ist die Wahrscheinlichkeit von Interferenzfehlern mit dem Deutschen gegeben. Zudem scheint die Wahrscheinlichkeit der Spiegelung der Mündlichkeit in der Schriftproduktion umso höher, wenn das Russische nur in der Familie gesprochen und im schriftlichen Bereich nicht verwendet wird.

Es ist jedoch offensichtlich, dass das Erheben von Metadaten nicht immer möglich ist oder deren Interpretation für die Analyse der Schriftproben sich als schwierig erweist. In diesem Fall können Erkenntnisse aus den bereits erwähnten Studien von Anstatt (2008, 2009, 2010, 2011), Bergmann (2014), Böhmer (2015), Brüggemann (2010, 2012, 2014), Dieser (2013), Karl (2012), Meng

Anka Bergmann, Olga Caspers \& Wolfgang Stadler (Hg.)

Didaktik der slawischen Sprachen - Beiträge zum 1. Arbeitskreis in Berlin (12.-14.9.2016)

(C) 2018 innsbruck university press, ISBN 978-3-903187-11-5, DOI 10.15203/3187-11-5 
(2001) und Rethage (2012) herangezogen werden.

\subsubsection{Zweiter Schritt - Kriterien erarbeiten}

„Die interessante Debatte zeigt: Ein Grundproblem jeder Textevaluation ist die Frage, welche Kriterien angelegt werden sollen", schreibt Fix (2008: 194) und verweist dabei auf Nussbaumer (1996: 96-112), der in seinem „Textanalyseraster“ zwischen den zwei Grundkategorien „sprachsystematische Richtigkeit“ und „Angemessenheit“ unterscheidet. Damit wird der Versuch unternommen, die oberflächenbezogene bzw. sprachformale Ebene von der tiefenstrukturellen bzw. konzeptionellen Ebene abzugrenzen. Diese Trennung entspricht auch der Sichtweise der Autorin dieses Artikels und wird des Weiteren übernommen. Zu beachten ist dabei, dass die Kategorien „Oberfläche“ und „Tiefe“ im Rahmen jedes einzelnen Textes schwer voneinander abgrenzbar sind (Fix 2008: 197). Die Zuordnung von Streitfällen zu der einen oder der anderen Kategorie liegt dementsprechend jedes Mal in den Händen der PrüferInnen und ist kontextabhängig. Dabei können neben dem Kontext selbst die erhobenen Metadaten sehr hilfreich sein, denn diese helfen die fehlerprovozierenden Mechanismen klar zu erkennen, was bei der Kategorisierung und Einordnung dieser Fehler hilft. Als Beispiel kann an dieser Stelle der häufig vorkommende Fehler genannt werden, wenn in einer unbetonten Endung $a$ statt $o$ geschrieben wird. Dieser Fehler kann verschiedene Gründe bzw. unterschiedliche fehlerprovozierende Mechanismen haben: Er kann als Rechtschreibfehler identifiziert werden, wenn aus den Metadaten hervorgeht, dass der/die Studierende Russisch nicht im institutionellen Kontext gelernt hat und deshalb ihm bzw. ihr der Bezug zwischen dem Gesprochenen und Geschriebenen fehlt. Dies kann aber auch ein Grammatikfehler sein, wenn wir aus den Metadaten wissen, dass er/sie einen Russischkurs besucht hat und die Endungen im Rahmen dessen gelernt hat. Bei unserer Bewertung wiegen solche Fehler unterschiedlich und dementsprechend werden für die Planung des Unterrichts die Schwerpunkte (in diesem Fall Orthographie oder Grammatik) auch unterschiedlich gesetzt, um diesen Fehler zu beheben.

Ein weiterer wichtiger Punkt sind die Anforderungen an die Bewertungskriterien. An diese „werden die gleichen Anforderungen gerichtet wie an wissenschaftliche Tests:

Anka Bergmann, Olga Caspers \& Wolfgang Stadler (Hg.)

Didaktik der slawischen Sprachen - Beiträge zum 1. Arbeitskreis in Berlin (12.-14.9.2016)

(C) 2018 innsbruck university press, ISBN 978-3-903187-11-5, DOI 10.15203/3187-11-5 
- Validität (Gültigkeit: Wird wirklich das gemessen, was behauptet wird?)

- Reliabilität (Zuverlässigkeit: Ist die Bewertung jederzeit mit gleichem Ergebnis wiederholbar?)

- Objektivität (Unabhängigkeit von subjektiven Einschätzungen der bewertenden Personen)“ (Fix 2008: 197).

Für die Validität und Reliabilität sieht u. a. Fix (2008: 199) zwei entscheidende Faktoren: den Grad der Routine der BewerterInnen sowie den Umfang des Kriterienrasters, wobei die beiden Faktoren direkt miteinander verbunden sind. Je unerfahrener die Lehrkraft ist, desto detaillierter sollte das Bewertungsraster sein bzw. desto mehr verlässt sie sich auf das Raster. Je höher die Anzahl der Kriterien im Raster, desto weniger valide und reliabel erweist es sich. Das beweisen Studien, die in Fix (2008) ausführlich diskutiert werden. Außerdem muss bezüglich des Umfangs des Rasters angemerkt werden, dass, je detaillierter dieses ist, desto zeitintensiver ist seine Anwendung. Im Rahmen eines Einstufungsverfahrens sind aufgrund der zeitlichen Knappheit umfangreiche Raster eher ungeeignet und wenig effizient.

Ein Aspekt der Auswertungsobjektivität ist bei der Einstufung von Studierenden einfach zu befolgen, wenn es darum geht, dass die Lehrkraft nicht von der Sympathie bzw. Antipathie zum/zur TeilnehmerIn beeinflusst wird. In unserem Fall ist dies kaum möglich, da sich Studierende und PrüferInnen nicht kennen.

Kommen wir nun zu den Kriterien selbst und betrachten wir zuerst die Fehler an der Textoberfläche. Zur sprachsystematischen Inkorrektheit zählen wir Grammatik-, Lexik-, Rechtschreib- und Interpunktions- sowie syntaktische Fehler, da ihr Erkennen keine tiefere Analyse der Struktur des Textes benötigt. Diese Fehler sind aus förderungsorientierter Perspektive von Wichtigkeit, da die erkannten Defizite zukünftig die Schwerpunkte des Unterrichts darstellen.

Zur Analyse solcher Fehler existieren bereits zahlreiche Publikationen, die jedoch von unterschiedlichen Lernertypen ausgehen. Kostomarov \& Mitrofanova (1984) klassifizieren typische Fehler von Russisch-als-Fremdsprache-Lernenden. Diese Klassifikation wurde von vielen WissenschaftlerInnen erweitert, so u. a. von Balychina \& Ignat'eva (2006), die auf unterschiedlichen sprachli-

Anka Bergmann, Olga Caspers \& Wolfgang Stadler (Hg.)

Didaktik der slawischen Sprachen - Beiträge zum 1. Arbeitskreis in Berlin (12.-14.9.2016)

(C) 2018 innsbruck university press, ISBN 978-3-903187-11-5, DOI 10.15203/3187-11-5 
chen Ebenen mehrere Fehlertypen beschreiben, welche auf zwei Hauptgründen basieren bzw. aufgrund von zwei fehlerprovozierenden Mechanismen passieren: Entweder liegt diesen Fehlern Interferenz zu Grunde (z. B. unterschiedliches Genus in der L1 und L2 kann zu Verwechslungen führen wie мой mempadz, эma график, этот уель) oder es handelt sich um sog. „Fehler des Niveaus“ (ошибки уровня). Bei letzteren handelt es sich um Fehler, die Lernende machen, weil sie bestimmte Strukturen noch nicht erworben bzw. gelernt haben. Diese Art von Fehlern wäre im Falle der Studierenden mit russischsprachigem Hintergrund irrelevant, da sie Russisch in den wenigsten Fällen institutionell gelernt haben und ihr Sprachniveau in der Regel nicht nach den Kompetenzniveaustufen des GeR (2001) gemessen werden kann. Jedoch kommen solche Fehler häufig vor. Die Lernenden mit russischsprachigem Hintergrund handeln meistens intuitiv und spontan, versuchen zu raten, bilden Strukturen entweder analog zum Deutschen - somit entstehen Interferenzfehler - oder sie orientieren sich an bekannten russischen Strukturen - und es kommt zu einer Übergrammatikalisierung. Eine andere Fehlerklassifikation, die in ein Bewertungsraster einfließen könnte, bietet die Didaktik des Russischen als Muttersprache. An dieser Stelle ist ein nach wie vor lesenswerter Beitrag von Zejtlin (1982) nennenswert, der in der russischen Fachliteratur auch immer noch zitiert wird. Zejtlin zeigt drei fehlerprovozierende Mechanismen auf und unterteilt mögliche Fehler in einer schriftlichen Produktion demensprechend in drei Gruppen (Zejtlin 1982: 7-12):

- „Fehler des Systems“ (системные оиибки), die auch als Fehler aufgrund der Übergrammatikalisierung bezeichnet werden können, z. B. durch „Füllung der Leerstellen“ (заполенение пустьхх клеток) im Paradigma (я пьлесосю, много мечт) oder durch „Anpassung“ von Ausnahmen der Regeln bzw. Übergeneralisierungen ( $я$ ездию). Solche Fehler, wie oben bereits angemerkt, sind auch für LernerInnen mit russischsprachigem Hintergrund typisch, kommen aber bei diesen noch häufiger als bei MuttersprachlerInnen mit Deutsch als L1 vor. Grund dafür ist, dass sie ,Leerstellen`im eigenen Sprachsystem füllen und unbekannte Wörter oder ganze Strukturen, die im Russischen zwar vorhanden, ihnen aber nicht bekannt sind, neu

Anka Bergmann, Olga Caspers \& Wolfgang Stadler (Hg.)

Didaktik der slawischen Sprachen - Beiträge zum 1. Arbeitskreis in Berlin (12.-14.9.2016)

(C) 2018 innsbruck university press, ISBN 978-3-903187-11-5, DOI 10.15203/3187-11-5 
bilden.

- Fehler, die durch den Einfluss der Mündlichkeit auf die Schriftlichkeit (просторечныле ошибки, v. a. auf der lexikalischen Ebene - ихний etc.) verursacht werden.

- „Kompositionsfehler“ (композичиннызе ошибки), die - nach Fix (2008) auch als Fehler der tiefenstrukturellen bzw. konzeptionellen Ebene bezeichnet werden können und aufgrund der Komplexität der Sprache oder fehlender Kenntnis bestimmter Textsorten geschehen können.

Da die Studierenden mit russischsprachigem Hintergrund nicht direkt einem der beiden genannten Lernertypen (Russisch-als-Fremdsprache-LernerInnen, MuttersprachlerInnen) zugeordnet werden können und einen besonderen Lernertyp bilden, muss dies auch im Kriterienraster für die Bewertung ihrer Schriftproben Beachtung finden. Eine solche Fehleranalyse, die als Basis für ein Kriterienraster zur Bewertung von Texten, verfasst von Studierenden mit russischsprachigem Hintergrund, dienen kann, wurde von Ermakova (2015) vorgeschlagen und soll an dieser Stelle kurz zusammengefasst und erweitert werden. Dieses Konzept vereint - nach dem Modell von Fix (2008) - Fehler, die einerseits das Sprachsystem betreffen und andererseits Fehler, die zur sprachlichen Unangemessenheit zählen.

- Graphische und graphomotorische Fehler: Am häufigsten zählt dazu die Verwechslung des lateinischen und kyrillischen Alphabets - pückar коnституиия.

- Orthographische Fehler: Diese passieren meistens aufgrund des Fehlens der Kenntnisse von Laut-Graphem-Korrespondenz im Russischen, manchmal aber auch aufgrund von Interferenzen - kaoc statt xaoc.

- Interpunktionsfehler: Hier sind zwei Tendenzen zu beobachten: Fehlende Interpunktion z. T. in Anlehnung an das Deutsche, in dem deutlich weniger Kommata als im Russischen benutzt werden oder überflüssige Interpunktion, wobei die Studierenden dort die Satzzeichen platzieren, wo sie beim Sprechen eine Pause machen. Eine besondere Schwierigkeit stellt z. B. die Satzzeichensetzung bei der indirekten (fehlerhaft mit einem Fragezeichen am Ende) und direkten Rede (fehlerhaft eingeführt) dar. 
- Morphologische Fehler: Gründe dafür können sowohl in der Interferenz liegen (своя суверенитет, настоящая время, новая этапа; кониентрируготся на проблему, получать помощь у государства) als auch in den lückenhaften Grammatikkenntnissen bzw. der Übergrammatikalisierung (2азеma продавается, это катастрофально).

- Lexikalische Fehler: Diese Kategorie kann zu den oberflächlichen Fehlern gehören (z. B. Neubildungen nach bekannten Mustern von Wörtern, die es nicht gibt - dazu könnte auch das oben genannte Wort „катастрофально“ zählen - oder aufgrund einer Interferenz - аттрактивный человек, он nрофитирует), aber auch zu Fehlern der tieferen Textebene. Im letzteren Fall kommt es zu Wiederholungen von Lexemen sowohl im Rahmen eines Satzes als auch zu lexikalischer Armut im ganzen Text, in dem die TypeToken-Relation sehr niedrig ist. Dies kann aber im Rahmen des Einstufungsverfahrens aufgrund der Zeitknappheit nur grob eingeschätzt werden.

- Fehler an der Textoberfläche: Damit ist gemeint, ob der Text in sich schlüssig, logisch und entsprechend gegliedert ist. Es muss dabei auch die Frage beantwortet werden, ob und wie, d. h. mit welchen sprachlichen Mitteln, die Textabschnitte miteinander verknüpft sind.

- Fehler der Textsortenangemessenheit: Dieser Punkt ist unmittelbar mit dem vorherigen Punkt verbunden: Entsprechen Form und Inhalt des Textes den Anforderungen der Textsorte? Konkreter heißt das: Sind die sprachlichen und strukturellen Merkmale dieser Textsorte in der Schriftprobe zu finden? Wurde das kommunikative Ziel des Textes erreicht?

\subsubsection{Dritter Schritt - Kriterien anwenden}

Bisher wurde viel über Fehler gesprochen. Das Wichtigste bei der Einstufung von Studierenden ist aber, nicht nur die Defizite zu erkennen, sondern die Vorkenntnisse, d. h., z. B. bereits erlernte und beherrschte grammatische Kategorien festzustellen. Dies sollte bei der Einstufung nicht vergessen werden, sodass das Potenzial des/der Lernenden nicht hinter den roten Korrekturen und Markierun- 
gen im Text verblasst.

Mne panravilas pervaja kartjinna, patamu sto ludje tam imejit bolshestvo haroshaju nastrajenju. fa vizju chelaveki, katoriji smishno tancujet ilji podgatovajit jedu. Mne bi tozje hotelarj paprobuvatj tortu, nu mnie kazujetsa, sto jedam tortu ja vigju solj i perec?

Ia njemagu ponjatj, u kamu djen rozjednija, morjit bitj, sto u maljecku, watorij sedjit na jesnici? Ot jevu vidnu potshti tolvo nos i glasa, patamu mne ne irvertna - eta djen rozjednija u jivo ilji u kavo ta druquvo? Ja vizju okolo 14 suechri, nu rebetji vigladjit chut pa staxshe.

Djeunshka, katoraja sedjit na polu, vigladjet neshislivaja, mothit bitj shto iz za parachko, katoriji celavajetsa.

Folgendes Beispiel (Abb. 1) soll der Erläuterung dieses Sachverhalts dienen:

Abbildung 1: Beispiel einer freien Schriftprobe

Die Teilnehmerin, die den abgebildeten Text verfasst hat, ist im Russischen nicht alphabetisiert, sie kann die kyrillische Schrift weder lesen noch schreiben. Jedoch wäre ein Anfängerkurs, in dem man zunächst Lesen und Schreiben lernt, für sie ungeeignet, denn sie verfügt über die meisten Wörter und grammatischen Strukturen, die in einem A1-Kurs gelehrt werden.

Die Metadaten dieser Probandin helfen, ihre Situation sowie die Besonderheiten ihres Textes zu verstehen. Sie kam im Vorschulalter nach Deutschland und hat seitdem kein Russisch im gesteuerten Kontext gelernt. Mit den in Russland gebliebenen Verwandten telefoniert und skypt sie auf Russisch, der Kontakt ist aber unregelmäßig. Die Eltern sprechen mit ihr meistens Russisch, sie antwortet aber häufiger auf Deutsch. Bevor sie sich entschied, ein philologisches Studium aufzunehmen, hatte sie wenig mit ihrer Herkunftssprache zu tun. 
Wenn wir von der lateinischen Schrift dieses Textes absehen, stellen wir fest, dass diese Studentin vielseitige Vorkenntnisse im Russischen hat. Sie kann bspw. argumentieren (мне понравилась первая картинка, потому ито... ${ }^{3}$ ), komplexe Sätze bilden (Я не могу понать, у кому день рождения, может бъгть, ито у мальчику, который сидит на лестниче?) und sie benutzt den Konjunktiv (Мне бы тоже хотелось попробоватв...). Sie verfügt über alltagsbezogenen Wortschatz, ihre Beherrschung ist aber stark von deutschen Interferenzen geprägt. So wird z. B. das Wort „настроение“ (die Stimmung, die Laune) dem femininen Paradigma, das Wort „nарочка“ (das Pärchen) dem Neutrum zugeordnet. Die vorhandenen vielseitigen, aber unstrukturierten Grammatikkenntnisse zeigen sich auch bei anderen Wortarten: Nach der Präposition y folgt manchmal der Dativ (у кому день рождения, у мальчику; analog der deutschen Konstruktion „bei wem“), manchmal der Genitiv (у него или у кого-то другого), sо wie im Russischen üblich. Die Konjugation der Verben ist meistens richtig: мне кажется; не могу понять; я вижу; девушка, которая сидит на полу, выллядит..., in anderen, vergleichbaren Kontexten jedoch falsch: человеки, которые таниует или подготоваит. Lassen wir den Gesamteindruck dieses Textes auf uns wirken, so erfüllt dieser zwar seine kommunikative Aufgabe (wir können uns das beschriebene Bild vorstellen, der Text ist logisch, gut strukturiert und unterhaltsam), er weist aber keine schriftsprachliche Korrektheit hinsichtlich Wortwahl, Satzbau und Interpunktion auf: ... мне неизвестно - это день рождения у него или у кого-то другого? Я вижу около 14 свечки, ну ребята выглядит чуть постарие. All das spricht dafür, dass wir es mit der Verschriftlichung (eher mit dem Versuch einer Verschriftlichung) eines mündlichen Textes zu tun haben.

Die orthographischen Fehler müssen an dieser Stelle nicht weiter diskutiert werden: Da die Kandidatin die kyrillische Schrift nicht beherrscht, fehlt ihr jede Vorstellung von Laut-Graphem-Korrelationen. In anderen Bereichen weist sie vielseitige, aber unstrukturierte Kenntnisse auf.

3 Die Textausschnitte aus der abgebildeten Schriftprobe werden des Weiteren in kyrillischer Schrift und mit der orthographischen Korrektur angeführt.

Anka Bergmann, Olga Caspers \& Wolfgang Stadler (Hg.)

Didaktik der slawischen Sprachen - Beiträge zum 1. Arbeitskreis in Berlin (12.-14.9.2016)

(C) 2018 innsbruck university press, ISBN 978-3-903187-11-5, DOI 10.15203/3187-11-5 


\section{Fazit}

Dank der im Einstufungstest am Zessko der Universität Potsdam geforderten freien Schriftproduktion können sowohl die Schwächen als auch die Stärken der ProbandInnen im Russischen festgestellt werden. Auch viele andere Texte der TeilnehmerInnen des Einstufungstests zeigen in erster Linie das Potenzial der VerfasserInnen. Die Lehrkräfte müssen diese Erkenntnisse als Ausgangspunkt für weitere Förderaktivitäten wahrnehmen und das Potenzial der StudentInnen nutzen. Die Anwendung der vorgeschlagenen Fehlerkriterien erleichtert nicht nur das Erkennen und Systematisieren von Problembereichen, sondern auch das Erfassen von Stärken. Wie die erfassten Stärken und Schwächen der beschriebenen Zielgruppe im Unterricht beachtet werden können, muss zukünftig ausführlich diskutiert und analysiert werden. Dabei wären vor allem weitere empirische Erhebungen eine große Hilfe.

\section{Literaturverzeichnis}

Anstatt, T. (2008). Russisch in Deutschland: Entwicklungsperspektiven. Bulletin der deutschen Slavistik, 14, 67-74.

Anstatt, T. (2009). Der Erwerb der Familiensprache: Zur Entwicklung des Russischen bei bilingualen Kindern in Deutschland. In I. Gogolin \& U. Neumann (Hrsg.), Streitfall Zweisprachigkeit - The Bilingualism Controversy (111-131). Wiesbaden: Springer VS.

Anstatt, T. (2009). Sprachattrition. Abbau der Erstsprache bei russisch-deutschen Jugendlichen. Wiener Slawistischer Almanach, 67, 7-31.

Anstatt, T. (2010). Kognitive Strategien Zweisprachiger. Lösungen lexikalischer Probleme im Russischen bilingualer Kinder und Jugendlicher. In T. Anstatt \& B. Norman (Hrsg.), Die slavischen Sprachen im Licht der kognitiven Linguistik / Славянские язики в свете когнитивной мингистики (217-239). Wiesbaden: Harrassowitz.

Anka Bergmann, Olga Caspers \& Wolfgang Stadler (Hg.)

Didaktik der slawischen Sprachen - Beiträge zum 1. Arbeitskreis in Berlin (12.-14.9.2016)

(C) 2018 innsbruck university press, ISBN 978-3-903187-11-5, DOI 10.15203/3187-11-5 
Anstatt, T. (2011). Russisch in der zweiten Generation. Zur Sprachsituation von Jugendlichen aus russischsprachigen Familien in Deutschland. In L. M. Eichinger, A. Plewnia \& M. Steinle (Hrsg.), Sprache und Integration. Über Mehrsprachigkeit und Integration (101-128). Tübingen: Narr.

Balychina, T. M. \& Ignat'eva, O. P. (2006). Аингводидактическая теория ошибки и пути преодоления ошибок в речи иностранньцх учашихся: Учеб. пособие. Moskva: RUDN.

Bergmann, A. (2014). О языковой и образовательной ситуации русскоязычных школьников в Германии. In A. Nikunlassi \& E. Protassova (Hrsg.), Ошибки и многоязычие (116-136). Helsinki: University of Helsinki.

Böhmer, J. (2015). Biliteralität: Eine Studie zu literaten Strukturen in Sprachproben von Jugendlichen im Deutschen und im Russischen. Münster: Waxmann.

Brüggemann, N. (2010). Сопоставительная мингвистика и преподавание грамматики русского языка в ситуации русско-немецкого двуязычия. In M. Remneva, A. Polikarpov \& O. Kukuškina (Hrsg.), Русский язык: исторические судьбъг и совеременность. Труды и материаль (590-591). Moskva: Izdatel'stvo Moskovskogo universiteta.

Brüggemann, N. (2012). Преподавание русского языка второму поколенио русскоязычныгх мигрантов: задачи, ироблемы, методические решения. Thesen zum Vortrag an der Staatlichen Universität Sankt-Petersburg.

Brüggemann, N. (2014). Herkunftssprache Russisch - unvollständige Grammatik als Folge mündlichen Spracherwerbs. In B. Sonnenhauser (Hrsg.), Linguistische Beiträge zur Slavistik. Materialien des Treffens der JungslavistInnen in München 2013 (37-58). München u. a.: BiblionMedia.

Dieser, E. (2013). Genuserwerb im Russischen und Deutschen. Korpusgestützte Studie zu ein-und zweisprachigen Kindern und Erwachsenen. München, Berlin: Verlag Otto Sagner.

Ermakova, N. (2014). К проблеме преподавания РКИ немекоговорящим студентам с русским / русскоязычным фоном. Русский язык за рубежом, 5, 39-43.

Ermakova, N. (2015). Ошибки в письменной речи немецкоговорящих студентов с русским / русскоязычным фоном. Русский язык за рубежом, 2, 42-46.

Ermakova, N. (2016). Особенности преподавания русского языка немецким студентамфилологам с русскоязычным фоном: этап вступительного тестирования и организации курсов. In M. Rusezkaja et al. (Hrsg.), Филологическое образование в современных исследованиях: мингвистический и методиеский аспекты. Материалы межАународной научно-практической конференции «САавянская культура: истоки, традиции, взаимодействие». XVII КирилмоМефодиевские чтения» (321-326). Moskva-Jaroslavl': Remder.

Fix, M. (2008). Texte schreiben. Schreibprozesse im Deutschunterricht. Paderborn: Schöningh. 
GeR=Trim, J., North, B. \& Coste, D. (Hrsg.) (2001). Gemeinsamer europäischer Referenzrahmen für Sprachen: Lernen, lehren, beurteilen. Berlin: Langenscheidt.

Karl, K. B. (2012). Bilinguale Lexik. Nicht materieller lexikalischer Transfer als Folge der aktuellen russisch-deutschen Zweisprachigkeit. München, Berlin: Verlag Otto Sagner.

Kostomarov, V. G. \& Mitrofanova, O. D. (1984). Методическое руководство для преподавателей русского языка иностраниам. Moskva: Russkij Jazyk.

Meng, K. (2001). Russlanddeutsche Sprachbiografien: Untersuchungen zur sprachlichen Integration von Aussiedlerfamilien. Tübingen: Narr.

Nussbaumer, M. (1996). Lernerorientierte Textanalyse - Eine Hilfe zum Textverfassen? In H. Feilke \& P. Portmann (Hrsg.), Schreiben im Umbruch. Schreibforschung und schulisches Schreiben (96-112). Stuttgart: Klett.

Reich, H. H. (2009). Aufbauende Sprachförderung unter Nutzung der FörMig-Instrumente. In D. Lengyel, H. H. Reich, H.-J. Roth \& M. Döll (Hrsg.), Von der Sprachdiagnose zur Sprachförderung (25-34). Münster u. a.: Waxmann.

Rethage, W. (2012). Strukturelle Besonderheiten des Russischen in Deutschland: kontaktlinguistische und soziolinguistische Aspekte. München, Berlin: Verlag Otto Sagner.

Schründer-Lenzen, A. \& Henn, D. (2009). Entwicklung eines Computerprogramms zur Analyse der schriftlichen Erzählfähigkeit. In D. Lengyel, H. H. Reich, H.-J. Roth, M. Döll (Hrsg.), Von der Sprachdiagnose zur Sprachförderung (91-108). Münster u. a.: Waxmann.

Zejtlin, S. N. (1982). Речевые очибки и их предупреждение. Moskva: Prosveščenie. 
